\title{
A NEW CLASS OF GENERATING FUNCTIONS OF BINARY PRODUCTS OF GAUSSIAN NUMBERS AND POLYNOMIALS
}

\author{
Souhila BOUGHABA, Ali BOUSSAYOUD, and Mohamed KERADA
}

LMAM Laboratory and Department of Mathematics, Mohamed Seddik Ben Yahia University, Jijel, ALGERIA

\begin{abstract}
In this paper, we introduce a operator in order to derive some new symmetric properties of Gaussian Fibonacci numbers. By making use of the operator defined in this paper, we give some new generating functions for Gaussian Fibonacci numbers, Gaussian Lucas numbers, Gaussian Pell numbers, Gaussian Pell Lucas numbers, Gaussian Jacobsthal numbers, Gaussian Jacobsthal polynomials, Gaussian Jacobsthal Lucas polynomials and Gaussian Pell polynomials.
\end{abstract}

\section{INTRODUCTION}

In the paper 9, 10], a second-order linear recurrence sequence $\left(U_{n}(a, b ; p, q)\right)_{n \geq 0}$ or briefly $\left(U_{n}\right)_{n \geq 0}$ is considered by the recurrence relation:

$$
U_{n+2}=p U_{n+1}+q U_{n},
$$

with the initial conditions $U_{0}=a$ and $U_{1}=b$, where $a, b \in \mathbb{C}$ and $p, q \in \mathbb{Z}_{+}$for $n \geq 0$. The special cases are listed below:

- For $p=q=b=1, a=i$ one gets the Gaussian Fibonacci numbers;

- For $p=q=1, a=2-i, b=1+2 i$ one has the Gaussian Lucas numbers;

- For $p=2, q=1, a=i, b=1$ one has the Gaussian Pell numbers;

- For $p=2, q=1, a=2-2 i, b=2+2 i$ one has the Gaussian Pell Lucas numbers;

- For $p=1, q=2, a=\frac{i}{2}, b=1$ it yields Gaussian Jacobsthal numbers;

- For $p=1, q=2, a=2-\frac{i}{2}, b=1+2 i$ one has the Gaussian Jacobsthal Lucas numbers.

2020 Mathematics Subject Classification. Primary 05E05; Secondary 11B39.

Keywords and phrases. Symmetric functions, generating functions, Gaussian Fibonacci numbers, Gaussian Lucas numbers, Gaussian Pell polynomials.

凶 souhilaboughaba@gmail.com-Corresponding author; aboussayoud@yahoo.fr; mkerada@yahoo.fr

(D) 0000-0002-5501-179X; 0000-0001-5553-3240; 0000-0002-0841-1555. 
In this paper, a second-order linear recurrence polynomials $P_{n}(x)$ is given by the following recurrence relation:

$$
P_{n+2}(x)=\alpha P_{n+1}(x)+\beta x P_{n}(x),
$$

with $P_{0}(x)=p+q x$ and $P_{1}(x)=r+s x$, where $p, q, r, s \in \mathbb{C}$ and $\alpha, \beta \in \mathbb{Z}_{+}$for $n \geq 0$. The special cases of the polynomials $P_{n}(x)$ are listed as follows:

- For $\alpha=r=1, \beta=2 ; p=\frac{i}{2}, q=s=0$ it yields the Gaussian Jacobsthal polynomials $G J_{n}(x)$;

- For $\alpha=1, \beta=2 ; p=2-\frac{i}{2}, q=0, r=1, s=2 i$ it reduces to the Gaussian Jacobsthal Lucas polynomials $G j_{n}(x)$.

In [11, Djordjevic and Srivastava defined incomplete generalized Jacobsthal and Jacobsthal-Lucas numbers.

The Gaussian Jacobsthal and Gaussian Jacobsthal Lucas polynomials $G J_{n}(x)$ and $G j_{n}(x)$ are defined and studied by authors 15 . They give generating function, Binet formula, explicit formula, $Q$ matrix, determinantal representations and partial derivation of these polynomials. S. Halici and S. Oz are defined in 2016 the Gaussian Pell and Gaussian Pell-Lucas numbers. They give generating functions and Binet formulas of Gaussian Pell and Gaussian Pell-Lucas numbers. The authors in 16 defined Gaussian Pell polynomials, they give the generating functions and Binet formulas for this type polynomials. On the other hand, many kinds of generalizations of Gaussian Fibonacci numbers have been presented in the literature. In particular, a generalization is the Gaussian Fibonacci numbers, the Gaussian Fibonacci numbers, say $\left(G F_{n}\right)_{n \in \mathbb{N}}$, is defined in [14, recurrently by

$$
\left\{\begin{array}{c}
G F_{0}=i, G F_{1}=1 \\
G F_{n}=G F_{n-1}+G F_{n-2}, \forall n \geq 2
\end{array} .\right.
$$

For $n>1$ : One can see that

$$
G F_{n}=F_{n}+i F_{n-1},
$$

where $F_{n}$ is the $n$-th usual Fibonacci numbers. by

The Gaussian Pell polynomials, say $\left(G P_{n}(x)\right)_{n \in \mathbb{N}}$, is defined in 16 recurrently

$$
\left\{\begin{array}{c}
G P_{0}(x)=i, G P_{1}(x)=1 \\
G P_{n}(x)=2 x G P_{n-1}(x)+G P_{n-2}(x), \forall n \geq 2
\end{array} .\right.
$$

It is note that we have an important relation between Gaussian Pell polynomials and usual Pell polynomials as follows.

$$
G P_{n}(x)=P_{n-1}(x)+i P_{n-2}(x), \quad \forall n \geq 2 .
$$

In this contribution, we shall define a useful operator denoted by $\delta_{a_{1} a_{2}}^{k}$ for which we can formulate, extend and prove new results based on our previous ones, (see [4 6]). In order to determine new generating functions of some well-known numbers 
and polynomials, we combine between our indicated past techniques and these presented polishing approaches.

In section 2, we introduce a symmetric function and give some properties of this symmetric function. We also give some more useful definitions which are used in the subsequent sections. we find generating functions of the products of Gaussian Fibonacci numbers, Gaussian Lucas numbers, Gaussian Pell numbers, Gaussian Pell Lucas numbers, Gaussian Jacobsthal numbers, Gaussian Jacobsthal polynomails, Gaussian Jacobsthal Lucas polynomails and Gaussian Pell polynomails, in section 3. In section 4 generating functions of some well-known polynomials.

\section{Definitions and some Properties}

In this section, we introduce a symmetric function and give some properties of this symmetric function. We also give some more useful definitions from the literature which are used in the subsequent sections.

We shall handle functions on different sets of indeterminates (called alphabets, though we shall mostly use commutative indeterminates for the moment). A symmetric function of an alphabet $A$ is a function of the letters which is invariant under permutation of the letters of $A$. Taking an extra indeterminate $z$, one has two fundamental series 3 .

$$
\lambda_{z}(A)=\Pi_{a \in A}(1+a z), \sigma_{z}(A)=\frac{1}{\Pi_{a \in A}(1-a z)},
$$

the expansion of which gives the elementary symmetric functions $\Lambda_{n}(A)$ and the complete functions $S_{n}(A)$ :

$$
\lambda_{z}(A)=\sum_{n=0}^{+\infty} \Lambda_{n}(A) z^{n}, \sigma_{z}(A)=\sum_{n=0}^{+\infty} S_{n}(A) z^{n} .
$$

Let us now start at the following definition.

Definition 1. (see [1]) Let $A$ and $B$ be any two alphabets, then we give $S_{n}(A-B)$ by the following form:

$$
\frac{\Pi_{b \in B}(1-b z)}{\Pi_{a \in A}(1-a z)}=\sum_{n=0}^{+\infty} S_{n}(A-B) z^{n}=\sigma_{z}(A-B),
$$

with the condition $S_{n}(A-B)=0$ for $n<0$.

Corollary 2. Taking $A=0$ in (2.1) gives

$$
\Pi_{b \in B}(1-b z)=\sum_{n=0}^{+\infty} S_{n}(-B) z^{n}=\lambda_{z}(-B) .
$$


Further, in the case $A=0$ or $B=0$, we have

$$
\sum_{n=0}^{+\infty} S_{n}(A-B) z^{n}=\sigma_{z}(A) \times \lambda_{z}(-B)
$$

Thus,

$$
S_{n}(A-B)=\sum_{k=0}^{n} S_{n-k}(A) S_{k}(-B)(\text { see [2] }) .
$$

Definition 3. [12] Let $n$ be positive integer and $A=\left\{a_{1}, a_{2}\right\}$ are set of given variables, then, the $n$-th symmetric function $S_{n}\left(a_{1}+a_{2}\right)$ is defined by

$$
S_{n}(A)=S_{n}\left(a_{1}+a_{2}\right)=\frac{a_{1}^{n+1}-a_{2}^{n+1}}{a_{1}-a_{2}},
$$

with

$$
\begin{aligned}
& S_{0}(A)=S_{0}\left(a_{1}+a_{2}\right)=1 \\
& S_{1}(A)=S_{1}\left(a_{1}+a_{2}\right)=a_{1}+a_{2}, \\
& S_{2}(A)=S_{2}\left(a_{1}+a_{2}\right)=a_{1}^{2}+a_{1} a_{2}+a_{2}^{2},
\end{aligned}
$$

Definition 4. 77 Given an alphabet $A=\left\{a_{1}, a_{2}\right\}$, the symmetrizing operator $\delta_{a_{1} a_{2}}^{k}$ is defined by

$$
\delta_{a_{1} a_{2}}^{k} f\left(a_{1}\right)=\frac{a_{1}^{k} f\left(a_{1}\right)-a_{2}^{k} f\left(a_{2}\right)}{a_{1}-a_{2}} .
$$

Example 5. If $f\left(a_{1}\right)=a_{1}$, the operator (2.5) gives us

$$
\delta_{a_{1} a_{2}}^{k} f\left(a_{1}\right)=\frac{a_{1}^{k+1}-a_{2}^{k+1}}{a_{1}-a_{2}}=S_{k}\left(a_{1}+a_{2}\right) .
$$

\section{Generating functions of some Well-Known numbers}

The following theorem is one of the key tools of the proof of our main result. It has been proved in 7$]$ for the completeness of the paper we state its proof here.

Theorem 6. Given two alphabets $A=\left\{a_{1}, a_{2}\right\}$ and $E=\left\{e_{1}, e_{2}\right\}$, then

$$
\sum_{n=0}^{+\infty} S_{n}(A) S_{n}(E) z^{n}=\frac{1-a_{1} a_{2} e_{1} e_{2} z^{2}}{\left(\sum_{n=0}^{+\infty} S_{n}(-A) e_{1}^{n} z^{n}\right)\left(\sum_{n=0}^{+\infty} S_{n}(-A) e_{2}^{n} z^{n}\right)} .
$$

Proof. By applying the operator $\delta_{e_{1} e_{2}}^{1}$ to the series $f\left(e_{1}\right)=\sum_{n=0}^{+\infty} S_{n}(A) e_{1}^{n} z^{n}$, the left hand side of formula (3.1) can be written as

$$
\delta_{e_{1} e_{2}}^{1} f\left(e_{1}\right)=\delta_{e_{1} e_{2}}^{1}\left(\sum_{n=0}^{+\infty} S_{n}(A) e_{1}^{n} z^{n}\right)
$$




$$
\begin{aligned}
& =\frac{e_{1} \sum_{n=0}^{+\infty} S_{n}(A) e_{1}^{n} z^{n}-e_{2} \sum_{n=0}^{+\infty} S_{n}(A) e_{2}^{n} z^{n}}{e_{1}-e_{2}} \\
& =\sum_{n=0}^{+\infty} S_{n}(A)\left(\frac{e_{1}^{n+1}-e_{2}^{n+1}}{e_{1}-e_{2}}\right) z^{n} \\
& =\sum_{n=0}^{+\infty} S_{n}(A) S_{n}(E) z^{n},
\end{aligned}
$$

and the right hand side of this formula can be written as

$$
\begin{gathered}
\delta_{e_{1} e_{2}}^{1}\left(\frac{1}{\sum_{n=0}^{+\infty} S_{n}(-A) e_{1}^{n} z^{n}}\right)=\frac{e_{1} \sum_{n=0}^{+\infty} S_{n}(-A) e_{2}^{n} z^{n}-e_{2} \sum_{n=0}^{+\infty} S_{n}(-A) e_{1}^{n} z^{n}}{\left(e_{1}-e_{2}\right)\left(\sum_{n=0}^{+\infty} S_{n}(-A) e_{1}^{n} z^{n}\right)\left(\sum_{n=0}^{+\infty} S_{n}(-A) e_{2}^{n} z^{n}\right)} \\
=\frac{e_{1}\left(1-a_{1} e_{2} z\right)\left(1-a_{2} e_{2} z\right)-e_{2}\left(1-a_{1} e_{1} z\right)\left(1-a_{2} e_{1} z\right)}{\left(e_{1}-e_{2}\right)\left(\sum_{n=0}^{+\infty} S_{n}(-A) e_{1}^{n} z^{n}\right)\left(\sum_{n=0}^{+\infty} S_{n}(-A) e_{2}^{n} z^{n}\right)} \\
=\frac{e_{1}\left(1-e_{2}\left(a_{1}+a_{2}\right) z+a_{1} a_{2} e_{2}^{2} z^{2}\right)-e_{2}\left(1-e_{1}\left(a_{1}+a_{2}\right) z+a_{1} a_{2} e_{1}^{2} z^{2}\right)}{\left(e_{1}-e_{2}\right)\left(\sum_{n=0}^{+\infty} S_{n}(-A) e_{1}^{n} z^{n}\right)\left(\sum_{n=0}^{+\infty} S_{n}(-A) e_{2}^{n} z^{n}\right)} \\
=\frac{1-a_{1} a_{2} e_{1} e_{2} z^{2}}{\left(\sum_{n=0}^{+\infty} S_{n}(-A) e_{1}^{n} z^{n}\right)\left(\sum_{n=0}^{+\infty} S_{n}(-A) e_{2}^{n} z^{n}\right)} .
\end{gathered}
$$

The proof is completed.

In this part, we now derive the new generating functions of the products of some known numbers.

For the case $A=\left\{a_{1},-a_{2}\right\}$ and $E=\left\{e_{1},-e_{2}\right\}$ with replacing $a_{2}$ by $\left(-a_{2}\right), e_{2}$ by $\left(-e_{2}\right)$ in $(3.1)$, we have

$\sum_{n=0}^{+\infty} S_{n}\left(a_{1}+\left[-a_{2}\right]\right) S_{n}\left(e_{1}+\left[-e_{2}\right]\right) z^{n}=\frac{1-a_{1} a_{2} e_{1} e_{2} z^{2}}{\left(1-a_{1} e_{1} z\right)\left(1+a_{2} e_{1} z\right)\left(1+a_{1} e_{2} z\right)\left(1-a_{2} e_{2} z\right)}$.

- Based on the relationship (3.2), we obtain

$$
\sum_{n=0}^{+\infty} S_{n-1}\left(a_{1}+\left[-a_{2}\right]\right) S_{n-1}\left(e_{1}+\left[-e_{2}\right]\right) z^{n}=\frac{z-a_{1} a_{2} e_{1} e_{2} z^{3}}{\left(1-a_{1} e_{1} z\right)\left(1+a_{2} e_{1} z\right)\left(1+a_{1} e_{2} z\right)\left(1-a_{2} e_{2} z\right)}
$$


This case consists of three related parts. Firstly, the substitutions

$$
\left\{\begin{array} { l } 
{ a _ { 1 } - a _ { 2 } = 1 } \\
{ a _ { 1 } a _ { 2 } = 2 }
\end{array} \text { and } \left\{\begin{array}{l}
e_{1}-e_{2}=1 \\
e_{1} e_{2}=2
\end{array}\right.\right.
$$

in (3.2) and (3.3), we obtain

$$
\begin{gathered}
\sum_{n=0}^{+\infty} S_{n}\left(a_{1}+\left[-a_{2}\right]\right) S_{n}\left(e_{1}+\left[-e_{2}\right]\right) z^{n}=\frac{1-4 z^{2}}{1-z-12 z^{2}-4 z^{3}+16 z^{4}}, \\
\sum_{n=0}^{+\infty} S_{n-1}\left(a_{1}+\left[-a_{2}\right]\right) S_{n-1}\left(e_{1}+\left[-e_{2}\right]\right) z^{n}=\frac{z-4 z^{3}}{1-z-12 z^{2}-4 z^{3}+16 z^{4}},
\end{gathered}
$$

from which we have the following theorems.

Theorem 7. For $n \in \mathbb{N}$, the new generating function of the product of Gaussian Jacobsthal numbers is given by

$$
\sum_{n=0}^{+\infty} G J_{n}^{2} z^{n}=\frac{-1+5 z+8(i+1) z^{2}-4(3-4 i) z^{3}}{4-4 z-48 z^{2}-16 z^{3}+64 z^{4}} .
$$

Proof. We know that

$$
G J_{n}=\left(\frac{i}{2} S_{n}\left(a_{1}+\left[-a_{2}\right]\right)+\left(1-\frac{i}{2}\right) S_{n-1}\left(a_{1}+\left[-a_{2}\right]\right)\right),(\text { see }[13]) .
$$

We see that

$$
\begin{aligned}
\sum_{n=0}^{+\infty} G J_{n}^{2} z^{n}= & \sum_{n=0}^{+\infty}\left(\frac{i}{2} S_{n}\left(a_{1}+\left[-a_{2}\right]\right)+\left(1-\frac{i}{2}\right) S_{n-1}\left(a_{1}+\left[-a_{2}\right]\right)\right) \\
& \times\left(\frac{i}{2} S_{n}\left(e_{1}+\left[-e_{2}\right]\right)+\left(1-\frac{i}{2}\right) S_{n-1}\left(e_{1}+\left[-e_{2}\right]\right)\right) z^{n} \\
= & \frac{-1}{4} \sum_{n=0}^{+\infty} S_{n}\left(a_{1}+\left[-a_{2}\right]\right) S_{n}\left(e_{1}+\left[-e_{2}\right]\right) z^{n} \\
+ & \left(\frac{i}{2}+\frac{1}{4}\right) \sum_{n=0}^{+\infty} S_{n}\left(a_{1}+\left[-a_{2}\right]\right) S_{n-1}\left(e_{1}+\left[-e_{2}\right]\right) z^{n} \\
+ & \left.\frac{i}{2}+\frac{1}{4}\right) \sum_{n=0}^{+\infty} S_{n}\left(e_{1}+\left[-e_{2}\right]\right) S_{n-1}\left(a_{1}+\left[-a_{2}\right]\right) z^{n} \\
+ & \left(1-\frac{i}{2}\right)^{2} \sum_{n=0}^{+\infty} S_{n-1}\left(a_{1}+\left[-a_{2}\right]\right) S_{n-1}\left(e_{1}+\left[-e_{2}\right]\right) z^{n} \\
= & \frac{-1+4 z^{2}}{4-4 z-48 z^{2}-16 z^{3}+64 z^{4}}+\frac{2(2 i+1)\left(z+2 z^{2}\right)}{4-4 z-48 z^{2}-16 z^{3}+64 z^{4}}
\end{aligned}
$$




$$
\begin{aligned}
& +\frac{(2-i)^{2}\left(z-4 z^{3}\right)}{4-4 z-48 z^{2}-16 z^{3}+64 z^{4}} \\
& =\frac{-1+5 z+8(i+1) z^{2}-4(3-4 i) z^{3}}{4-4 z-48 z^{2}-16 z^{3}+64 z^{4}} .
\end{aligned}
$$

This completes the proof.

Theorem 8. For $n \in \mathbb{N}$, the new generating function of the product of Gaussian Jacobsthal Lucas numbers is given by

$$
\sum_{n=0}^{+\infty} G j_{n}^{2} z^{n}=\frac{15-8 i+(24 i-27) z+(120 i-72) z^{2}+(80 i+84) z^{3}}{4-4 z-48 z^{2}-16 z^{3}+64 z^{4}}
$$

Proof. Since

$$
G j_{n}=\left(2-\frac{i}{2}\right) S_{n}\left(a_{1}+\left[-a_{2}\right]\right)+\left(\frac{5 i}{2}-1\right) S_{n-1}\left(a_{1}+\left[-a_{2}\right]\right), \quad(\text { see }[13) .
$$

From which we have

$$
\begin{aligned}
\sum_{n=0}^{+\infty} G j_{n}^{2} z^{n}= & \sum_{n=0}^{+\infty}\left(\left(2-\frac{i}{2}\right) S_{n}\left(a_{1}+\left[-a_{2}\right]\right)+\left(\frac{5 i}{2}-1\right) S_{n-1}\left(a_{1}+\left[-a_{2}\right]\right)\right) \\
& \times\left(\left(2-\frac{i}{2}\right) S_{n}\left(e_{1}+\left[-e_{2}\right]\right)+\left(\frac{5 i}{2}-1\right) S_{n-1}\left(e_{1}+\left[-e_{2}\right]\right)\right) z^{n} \\
= & \left(2-\frac{i}{2}\right)^{2} \sum_{n=0}^{+\infty} S_{n}\left(a_{1}+\left[-a_{2}\right]\right) S_{n}\left(e_{1}+\left[-e_{2}\right]\right) z^{n} \\
+ & \left(2-\frac{i}{2}\right)\left(\frac{5 i}{2}-1\right) \sum_{n=0}^{+\infty} S_{n}\left(a_{1}+\left[-a_{2}\right]\right) S_{n-1}\left(e_{1}+\left[-e_{2}\right]\right) z^{n} \\
+ & \left(2-\frac{i}{2}\right)\left(\frac{5 i}{2}-1\right) \sum_{n=0}^{+\infty} S_{n}\left(e_{1}+\left[-e_{2}\right]\right) S_{n-1}\left(a_{1}+\left[-a_{2}\right]\right) z^{n} \\
+ & \left(\frac{5 i}{2}-1\right)^{2} \sum_{n=0}^{+\infty} S_{n-1}\left(a_{1}+\left[-a_{2}\right]\right) S_{n-1}\left(e_{1}+\left[-e_{2}\right]\right) z^{n} .
\end{aligned}
$$

Then

$$
\begin{aligned}
\sum_{n=0}^{+\infty} G j_{n}^{2} z^{n} & =\frac{(15-8 i)\left(1-4 z^{2}\right)}{4-4 z-48 z^{2}-16 z^{3}+64 z^{4}}+\frac{(44 i-6)\left(z+2 z^{2}\right)}{4-4 z-48 z^{2}-16 z^{3}+64 z^{4}} \\
& -\frac{(20 i+21)\left(z-4 z^{3}\right)}{4-4 z-48 z^{2}-16 z^{3}+64 z^{4}} \\
& =\frac{15-8 i+(24 i-27) z+(120 i-72) z^{2}+(80 i+84) z^{3}}{4-4 z-48 z^{2}-16 z^{3}+64 z^{4}} .
\end{aligned}
$$

The proof is completed. 
Secondly, the substitutions

$$
\left\{\begin{array} { l } 
{ a _ { 1 } - a _ { 2 } = 1 } \\
{ a _ { 1 } a _ { 2 } = 1 }
\end{array} \text { and } \left\{\begin{array}{l}
e_{1}-e_{2}=1 \\
e_{1} e_{2}=1
\end{array},\right.\right.
$$

in (3.2) and (3.3) we obtain

$$
\begin{aligned}
\sum_{n=0}^{+\infty} S_{n}\left(a_{1}+\left[-a_{2}\right]\right) S_{n}\left(e_{1}+\left[-e_{2}\right]\right) z^{n} & =\frac{1-z^{2}}{1-z-4 z^{2}-z^{3}+z^{4}}, \\
\sum_{n=0}^{+\infty} S_{n-1}\left(a_{1}+\left[-a_{2}\right]\right) S_{n-1}\left(e_{1}+\left[-e_{2}\right]\right) z^{n} & =\frac{z-z^{3}}{1-z-4 z^{2}-z^{3}+z^{4}}
\end{aligned}
$$

We have the following theorems.

Theorem 9. For $n \in \mathbb{N}$, the new generating function of the product of Gaussian Fibonacci numbers is given by

$$
\sum_{n=0}^{+\infty} G F_{n}^{2} z^{n}=\frac{-1+2 z+(2 i+3) z^{2}+2 i z^{3}}{1-z-4 z^{2}-z^{3}+z^{4}} .
$$

Proof. From the reference 13] we have

$$
G F_{n}=i S_{n}\left(a_{1}+\left[-a_{2}\right]\right)+(1-i) S_{n-1}\left(a_{1}+\left[-a_{2}\right]\right),
$$

and from it

$$
\begin{aligned}
\sum_{n=0}^{+\infty} G F_{n}^{2} z^{n}= & \sum_{n=0}^{+\infty}\left(i S_{n}\left(a_{1}+\left[-a_{2}\right]\right)+(1-i) S_{n-1}\left(a_{1}+\left[-a_{2}\right]\right)\right) \\
& \times\left(i S_{n}\left(e_{1}+\left[-e_{2}\right]\right)+(1-i) S_{n-1}\left(e_{1}+\left[-e_{2}\right]\right)\right) z^{n} \\
= & -\sum_{n=0}^{+\infty} S_{n}\left(a_{1}+\left[-a_{2}\right]\right) S_{n}\left(e_{1}+\left[-e_{2}\right]\right) z^{n} \\
+ & i(1-i) \sum_{n=0}^{+\infty} S_{n}\left(a_{1}+\left[-a_{2}\right]\right) S_{n-1}\left(e_{1}+\left[-e_{2}\right]\right) z^{n} \\
+ & i(1-i) \sum_{n=0}^{+\infty} S_{n}\left(e_{1}+\left[-e_{2}\right]\right) S_{n-1}\left(a_{1}+\left[-a_{2}\right]\right) z^{n} \\
& (1-i)^{2} \sum_{n=0}^{+\infty} S_{n-1}\left(a_{1}+\left[-a_{2}\right]\right) S_{n-1}\left(e_{1}+\left[-e_{2}\right]\right) z^{n} .
\end{aligned}
$$

Therefore

$$
\sum_{n=0}^{+\infty} G F_{n}^{2} z^{n}=\frac{z^{2}-1}{1-z-4 z^{2}-z^{3}+z^{4}}+\frac{2(1+i)\left(z+z^{2}\right)}{1-z-4 z^{2}-z^{3}+z^{4}}
$$




$$
\begin{gathered}
+\frac{(1-i)^{2}\left(z-z^{3}\right)}{1-z-4 z^{2}-z^{3}+z^{4}} \\
=\frac{-1+2 z+(2 i+3) z^{2}+2 i z^{3}}{1-z-4 z^{2}-z^{3}+z^{4}} .
\end{gathered}
$$

This completes the proof.

Theorem 10. For $n \in \mathbb{N}$, the new generating function of the product of Gaussian Lucas numbers is given by

$$
\sum_{n=0}^{+\infty} G L_{n}^{2} z^{n}=\frac{3-4 i+(8 i-6) z+(18 i-1) z+(6 i+8) z^{3}}{1-z-4 z^{2}-z^{3}+z^{4}} .
$$

Proof. According to [13], we have

$$
G L_{n}=(2-i) S_{n}\left(a_{1}+\left[-a_{2}\right]\right)+(3 i-1) S_{n-1}\left(a_{1}+\left[-a_{2}\right]\right) .
$$

From which we have

$$
\begin{aligned}
\sum_{n=0}^{+\infty} G L_{n}^{2} z^{n}= & \sum_{n=0}^{+\infty}\left((2-i) S_{n}\left(a_{1}+\left[-a_{2}\right]\right)+(3 i-1) S_{n-1}\left(a_{1}+\left[-a_{2}\right]\right)\right) \\
& \times\left((2-i) S_{n}\left(e_{1}+\left[-e_{2}\right]\right)+(3 i-1) S_{n-1}\left(e_{1}+\left[-e_{2}\right]\right)\right) z^{n} \\
= & (2-i)^{2} \sum_{n=0}^{+\infty} S_{n}\left(a_{1}+\left[-a_{2}\right]\right) S_{n}\left(e_{1}+\left[-e_{2}\right]\right) z^{n} \\
+ & (2-i)(3 i-1) \sum_{n=0}^{+\infty} S_{n}\left(a_{1}+\left[-a_{2}\right]\right) S_{n-1}\left(e_{1}+\left[-e_{2}\right]\right) z^{n} \\
+ & (2-i)(3 i-1) \sum_{n=0}^{+\infty} S_{n}\left(e_{1}+\left[-e_{2}\right]\right) S_{n-1}\left(a_{1}+\left[-a_{2}\right]\right) z^{n} \\
& +(3 i-1)^{2} \sum_{n=0}^{+\infty} S_{n-1}\left(a_{1}+\left[-a_{2}\right]\right) S_{n-1}\left(e_{1}+\left[-e_{2}\right]\right) z^{n} .
\end{aligned}
$$

By using the relationships (3.6) and (3.7), we obtain

$$
\begin{aligned}
\sum_{n=0}^{+\infty} G L_{n}^{2} z^{n}= & \frac{(3-4 i)\left(1-z^{2}\right)}{1-z-4 z^{2}-z^{3}+z^{4}}+\frac{2(7 i+1)\left(z+z^{2}\right)}{1-z-4 z^{2}-z^{3}+z^{4}} \\
& +\frac{(3 i-1)^{2}\left(z-z^{3}\right)}{1-z-4 z^{2}-z^{3}+z^{4}} \\
= & \frac{3-4 i+(8 i-6) z+(18 i-1) z^{2}+(6 i+8) z^{3}}{1-z-4 z^{2}-z^{3}+z^{4}} .
\end{aligned}
$$

The proof is completed. 
Thirdly, the substitutions

$$
\left\{\begin{array} { l } 
{ a _ { 1 } - a _ { 2 } = 2 } \\
{ a _ { 1 } a _ { 2 } = 1 }
\end{array} \text { and } \left\{\begin{array}{l}
e_{1}-e_{2}=2 \\
e_{1} e_{2}=1
\end{array}\right.\right.
$$

in (3.2) and (3.3) we obtain

$$
\begin{gathered}
\sum_{n=0}^{+\infty} S_{n}\left(e_{1}+\left[-e_{2}\right]\right) S_{n}\left(e_{1}+\left[-e_{2}\right]\right) z^{n}=\frac{1-z^{2}}{1-4 z-10 z^{2}-4 z^{3}+z^{4}}, \\
\sum_{n=0}^{+\infty} S_{n-1}\left(e_{1}+\left[-e_{2}\right]\right) S_{n-1}\left(e_{1}+\left[-e_{2}\right]\right) z^{n}=\frac{z-z^{3}}{1-4 z-10 z^{2}-4 z^{3}+z^{4}},
\end{gathered}
$$

and we have the following theorems.

Theorem 11. For $n \in \mathbb{N}$, the new generating functions of the product of Gaussian Pell numbers is given by

$$
\sum_{n=0}^{+\infty} G P_{n}^{2} z^{n}=\frac{-1+5 z+(9+4 i) z^{2}+(3+4 i) z^{3}}{1-4 z-10 z^{2}-4 z^{3}+z^{4}} .
$$

Proof. By [13, we have $G P_{n}=i S_{n}\left(a_{1}+\left[-a_{2}\right]\right)+(1-2 i) S_{n-1}\left(a_{1}+\left[-a_{2}\right]\right)$.

Then, we can see that

$$
\begin{aligned}
\sum_{n=0}^{+\infty} G P_{n}^{2} z^{n}= & \sum_{n=0}^{+\infty}\left(i S_{n}\left(a_{1}+\left[-a_{2}\right]\right)+(1-2 i) S_{n-1}\left(a_{1}+\left[-a_{2}\right]\right)\right) \\
& \times\left(i S_{n}\left(e_{1}+\left[-e_{2}\right]\right)+(1-2 i) S_{n-1}\left(e_{1}+\left[-e_{2}\right]\right)\right) z^{n} \\
= & i^{2} \sum_{n=0}^{+\infty} S_{n}\left(a_{1}+\left[-a_{2}\right]\right) S_{n}\left(e_{1}+\left[-e_{2}\right]\right) z^{n} \\
+ & i(1-2 i) \sum_{n=0}^{+\infty} S_{n}\left(a_{1}+\left[-a_{2}\right]\right) S_{n-1}\left(e_{1}+\left[-e_{2}\right]\right) z^{n} \\
& +i(1-2 i) \sum_{n=0}^{+\infty} S_{n}\left(e_{1}+\left[-e_{2}\right]\right) S_{n-1}\left(a_{1}+\left[-a_{2}\right]\right) z^{n} \\
& +(1-2 i)^{2} \sum_{n=0}^{+\infty} S_{n-1}\left(a_{1}+\left[-a_{2}\right]\right) S_{n-1}\left(e_{1}+\left[-e_{2}\right]\right) z^{n}
\end{aligned}
$$

Therefore

$$
\begin{aligned}
\sum_{n=0}^{+\infty} G P_{n}^{2} z^{n}= & \frac{-1\left(1-z^{2}\right)}{1-4 z-10 z^{2}-4 z^{3}+z^{4}}+\frac{2 i(1-2 i)\left(2 z+2 z^{2}\right)}{1-4 z-10 z^{2}-4 z^{3}+z^{4}} \\
& +\frac{(1-2 i)^{2}\left(z-z^{3}\right)}{1-4 z-10 z^{2}-4 z^{3}+z^{4}}
\end{aligned}
$$




$$
=\frac{-1+5 z+(9+4 i) z^{2}+(3+4 i) z^{3}}{1-4 z-10 z^{2}-4 z^{3}+z^{4}} .
$$

This completes the proof.

Theorem 12. For $n \in \mathbb{N}$, the new generating functions of the product of Gaussian Pell Lucas numbers is given by

$$
\sum_{n=0}^{+\infty} G Q_{n}^{2} z^{n}=\frac{-8 i+40 i z+(32+72 i) z^{2}+(32+24 i) z^{3}}{1-4 z-10 z^{2}-4 z^{3}+z^{4}} .
$$

Proof. Since

$$
G Q_{n}=(2-2 i) S_{n}\left(a_{1}+\left[-a_{2}\right]\right)+(6 i-2) S_{n-1}\left(a_{1}+\left[-a_{2}\right]\right), \quad(\text { see }[13) .
$$

From which we have

$$
\begin{aligned}
\sum_{n=0}^{+\infty} G Q_{n}^{2} z^{n}= & \sum_{n=0}^{+\infty}\left((2-2 i) S_{n}\left(a_{1}+\left[-a_{2}\right]\right)+(6 i-2) S_{n-1}\left(a_{1}+\left[-a_{2}\right]\right)\right) \\
& \times\left((2-2 i) S_{n}\left(e_{1}+\left[-e_{2}\right]\right)+(6 i-2) S_{n-1}\left(e_{1}+\left[-e_{2}\right]\right)\right) z^{n} \\
= & (2-2 i)^{2} \sum_{n=0}^{+\infty} S_{n}\left(a_{1}+\left[-a_{2}\right]\right) S_{n}\left(e_{1}+\left[-e_{2}\right]\right) z^{n} \\
+ & (2-2 i)(6 i-2) \sum_{n=0}^{+\infty} S_{n}\left(a_{1}+\left[-a_{2}\right]\right) S_{n-1}\left(e_{1}+\left[-e_{2}\right]\right) z^{n} \\
+ & (2-2 i)(6 i-2) \sum_{n=0}^{+\infty} S_{n}\left(e_{1}+\left[-e_{2}\right]\right) S_{n-1}\left(a_{1}+\left[-a_{2}\right]\right) z^{n} \\
& +(6 i-2)^{2} \sum_{n=0}^{+\infty} S_{n-1}\left(a_{1}+\left[-a_{2}\right]\right) S_{n-1}\left(e_{1}+\left[-e_{2}\right]\right) z^{n} .
\end{aligned}
$$

Therefore

$$
\begin{aligned}
\sum_{n=0}^{+\infty} G Q_{n}^{2} z^{n}= & \frac{(2-2 i)^{2}\left(1-z^{2}\right)}{1-4 z-10 z^{2}-4 z^{3}+z^{4}}+\frac{2(2-2 i)(6 i-2)\left(2 z+2 z^{2}\right)}{1-4 z-10 z^{2}-4 z^{3}+z^{4}} \\
& +\frac{(6 i-2)^{2}\left(z-z^{3}\right)}{1-4 z-10 z^{2}-4 z^{3}+z^{4}} \\
= & \frac{-8 i+40 i z+(32+72 i) z^{2}+(32+24 i) z^{3}}{1-4 z-10 z^{2}-4 z^{3}+z^{4}} .
\end{aligned}
$$

The proof is completed. 


\section{Generating functions of some Well-Known polynomials}

In this part, we now derive the new generating functions of the products of some known polynomials.

This case consists of two related parts. Firstly, the substitutions

$$
\left\{\begin{array} { l } 
{ a _ { 1 } - a _ { 2 } = 1 } \\
{ a _ { 1 } a _ { 2 } = 2 x }
\end{array} \text { and } \left\{\begin{array}{l}
e_{1}-e_{2}=1 \\
e_{1} e_{2}=2 y
\end{array}\right.\right.
$$

in (3.2) and (3.3) we obtain

$$
\begin{array}{rl}
\sum_{n=0}^{+\infty} S_{n}\left(a_{1}+\left[-a_{2}\right]\right) S_{n}\left(e_{1}+\left[-e_{2}\right]\right) z^{n} & 1-4 x y z^{2} \\
& =\frac{1-2(x+y+4 x y) z^{2}-4 x y z^{3}+16 x^{2} y^{2} z^{4}}{1-z-2} \\
\sum_{n=0}^{+\infty} S_{n-1}\left(a_{1}\right. & \left.+\left[-a_{2}\right]\right) S_{n-1}\left(e_{1}+\left[-e_{2}\right]\right) z^{n} \\
& =\frac{z-4 x y z^{3}}{1-z-2(x+y+4 x y) z^{2}-4 x y z^{3}+16 x^{2} y^{2} z^{4}}
\end{array}
$$

from which we have the following theorems.

Theorem 13. For $n \in \mathbb{N}$, the new generating function of product of Gaussian Jacobsthal polynomials is given by

$$
\sum_{n=0}^{+\infty} G J_{n}(x) G J_{n}(y) z^{n}=\frac{-1+5 z+((4 i+2)(x+y)+4 x y) z^{2}-4 x y(3-4 i) z^{3}}{4-4 z-8(x+y+4 x y) z^{2}-16 x y z^{3}+64 x^{2} y^{2} z^{4}}
$$

Proof. From the reference [13] we have

$$
G J_{n}(x)=\frac{i}{2} S_{n}\left(a_{1}+\left[-a_{2}\right]\right)+\left(1-\frac{i}{2}\right) S_{n-1}\left(a_{1}+\left[-a_{2}\right]\right),
$$

and from it

$$
\begin{aligned}
\sum_{n=0}^{+\infty} G J_{n}(x) G J_{n}(y) z^{n}= & \sum_{n=0}^{+\infty}\left(\frac{i}{2} S_{n}\left(a_{1}+\left[-a_{2}\right]\right)+\left(1-\frac{i}{2}\right) S_{n-1}\left(a_{1}+\left[-a_{2}\right]\right)\right) \\
& \times\left(\frac{i}{2} S_{n}\left(e_{1}+\left[-e_{2}\right]\right)+\left(1-\frac{i}{2}\right) S_{n-1}\left(e_{1}+\left[-e_{2}\right]\right)\right) z^{n} \\
= & \frac{-1}{4} \sum_{n=0}^{+\infty} S_{n}\left(a_{1}+\left[-a_{2}\right]\right) S_{n}\left(e_{1}+\left[-e_{2}\right]\right) z^{n} \\
+ & \frac{i}{2}\left(1-\frac{i}{2}\right) \sum_{n=0}^{+\infty} S_{n}\left(a_{1}+\left[-a_{2}\right]\right) S_{n-1}\left(e_{1}+\left[-e_{2}\right]\right) z^{n}
\end{aligned}
$$




$$
\begin{aligned}
& +\quad \frac{i}{2}\left(1-\frac{i}{2}\right) \sum_{n=0}^{+\infty} S_{n}\left(e_{1}+\left[-e_{2}\right]\right) S_{n-1}\left(a_{1}+\left[-a_{2}\right]\right) z^{n} \\
& +\quad\left(1-\frac{i}{2}\right)^{2} \sum_{n=0}^{+\infty} S_{n-1}\left(a_{1}+\left[-a_{2}\right]\right) S_{n-1}\left(e_{1}+\left[-e_{2}\right]\right) z^{n} .
\end{aligned}
$$

Therefore

$$
\begin{aligned}
\sum_{n=0}^{+\infty} G J_{n}(x) G J_{n}(y) z^{n} & =\frac{4 x y z^{2}-1}{4-4 z-8(x+y+4 x y) z^{2}-16 x y z^{3}+64 x^{2} y^{2} z^{4}} \\
& +\frac{(2 i+1)\left(z+2 x z^{2}\right)}{4-4 z-8(x+y+4 x y) z^{2}-16 x y z^{3}+64 x^{2} y^{2} z^{4}} \\
& +\frac{(2 i+1)\left(z+2 y z^{2}\right)}{4-4 z-8(x+y+4 x y) z^{2}-16 x y z^{3}+64 x^{2} y^{2} z^{4}} \\
& +\frac{(3-4 i)\left(z-4 x y z^{3}\right)}{4-4 z-8(x+y+4 x y) z^{2}-16 x y z^{3}+64 x^{2} y^{2} z^{4}} \\
& =\frac{-1+5 z+((4 i+2)(x+y)+4 x y) z^{2}-4 x y(3-4 i) z^{3}}{4-4 z-8(x+y+4 x y) z^{2}-16 x y z^{3}+64 x^{2} y^{2} z^{4}} .
\end{aligned}
$$

This completes the proof.

Theorem 14. For $n \in \mathbb{N}$, the new generating function of product of Gaussian Jacobsthal Lucas polynomials is given by

$$
\begin{aligned}
& \sum_{n=0}^{+\infty} G j_{n}(x) G j_{n}(y) z^{n} \\
& =\frac{15-8 i+(8 i(x+y)-16 x y-11+8 i) z-((14-12 i)(x+y)+(44-96 i) x y) z^{2}}{4-4 z-8(x+y+4 x y) z^{2}-16 x y z^{3}+64 x^{2} y^{2} z^{4}} \\
& \quad+\frac{4 x y(16 x y+(4+8 i)(x+y)-(3-4 i)) z^{3}}{4-4 z-8(x+y+4 x y) z^{2}-16 x y z^{3}+64 x^{2} y^{2} z^{4}} .
\end{aligned}
$$

Proof. We know that

$$
G j_{n}(x)=\left(2-\frac{i}{2}\right) S_{n}\left(a_{1}+\left[-a_{2}\right]\right)+\left(2 i x+\frac{i}{2}-1\right) S_{n-1}\left(a_{1}+\left[-a_{2}\right]\right) \text {, (see [13]). }
$$

We see that

$$
\begin{aligned}
\sum_{n=0}^{+\infty} G j_{n}(x) G j_{n}(y) z^{n}= & \sum_{n=0}^{+\infty}\left(\left(2-\frac{i}{2}\right) S_{n}\left(a_{1}+\left[-a_{2}\right]\right)+\left(2 i x+\frac{i}{2}-1\right) S_{n-1}\left(a_{1}+\left[-a_{2}\right]\right)\right) \\
& \times\left(\left(2-\frac{i}{2}\right) S_{n}\left(e_{1}+\left[-e_{2}\right]\right)+\left(2 i y+\frac{i}{2}-1\right) S_{n-1}\left(e_{1}+\left[-e_{2}\right]\right)\right) z^{n} \\
= & \left(2-\frac{i}{2}\right)^{2} \sum_{n=0}^{+\infty} S_{n}\left(a_{1}+\left[-a_{2}\right]\right) S_{n}\left(e_{1}+\left[-e_{2}\right]\right) z^{n}
\end{aligned}
$$




$$
\begin{aligned}
& +\quad\left(2-\frac{i}{2}\right)\left(2 i y+\frac{i}{2}-1\right) \sum_{n=0}^{+\infty} S_{n}\left(a_{1}+\left[-a_{2}\right]\right) S_{n-1}\left(e_{1}+\left[-e_{2}\right]\right) z^{n} \\
& +\quad\left(2-\frac{i}{2}\right)\left(2 i x+\frac{i}{2}-1\right) \sum_{n=0}^{+\infty} S_{n}\left(e_{1}+\left[-e_{2}\right]\right) S_{n-1}\left(a_{1}+\left[-a_{2}\right]\right) z^{n} \\
& +\quad\left(2 i x+\frac{i}{2}-1\right)\left(2 i y+\frac{i}{2}-1\right) \\
& \quad \times \sum_{n=0}^{+\infty} S_{n-1}\left(a_{1}+\left[-a_{2}\right]\right) S_{n-1}\left(e_{1}+\left[-e_{2}\right]\right) z^{n} \\
& =\frac{\left(2-\frac{i}{2}\right)^{2}\left(1-4 x y z^{2}\right)}{1-z-2(x+y+4 x y) z^{2}-4 x y z^{3}+16 x^{2} y^{2} z^{4}} \\
& +\quad \frac{\left(2-\frac{i}{2}\right)\left(2 i y+\frac{i}{2}-1\right)\left(z+2 x z^{2}\right)}{1-z-2(x+y+4 x y) z^{2}-4 x y z^{3}+16 x^{2} y^{2} z^{4}} \\
& +\quad \frac{\left(2-\frac{i}{2}\right)\left(2 i x+\frac{i}{2}-1\right)\left(z+2 y z^{2}\right)}{1-z-2(x+y+4 x y) z^{2}-4 x y z^{3}+16 x^{2} y^{2} z^{4}} \\
& +\quad \frac{\left(2 i x+\frac{i}{2}-1\right)\left(2 i y+\frac{i}{2}-1\right)\left(z-4 x y z^{3}\right)}{1-z-2(x+y+4 x y) z^{2}-4 x y z^{3}+16 x^{2} y^{2} z^{4}} \\
& =\frac{15-8 i+(8 i(x+y)-16 x y-11+8 i) z-((14-12 i)(x+y)}{4-4 z-8(x+y+4 x y) z^{2}-16 x y z^{3}+64 x^{2} y^{2} z^{4}} \\
& +\quad \frac{(44-96 i) x y) z^{2}+4 x y(16 x y+(4+8 i)(x+y)-(3-4 i)) z^{3}}{4-4 z-8(x+y+4 x y) z^{2}-16 x y z^{3}+64 x^{2} y^{2} z^{4}}
\end{aligned}
$$

This completes the proof.

Secondly, the substitutions

$$
\left\{\begin{array} { c } 
{ a _ { 1 } - a _ { 2 } = 2 x } \\
{ a _ { 1 } a _ { 2 } = 1 }
\end{array} \text { and } \left\{\begin{array}{c}
e_{1}-e_{2}=2 y \\
e_{1} e_{2}=1
\end{array}\right.\right.
$$

in (3.2) and (3.3) we give

$$
\begin{aligned}
& \sum_{n=0}^{+\infty} S_{n}\left(a_{1}+\left[-a_{2}\right]\right) S_{n}\left(e_{1}+\left[-e_{2}\right]\right) z^{n}=\frac{1-z^{2}}{1-4 x y z-\left(4\left(x^{2}+y^{2}\right)+2\right) z^{2}-4 x y z^{3}+z^{4}} \\
& \sum_{n=0}^{+\infty} S_{n-1}\left(a_{1}+\left[-a_{2}\right]\right) S_{n-1}\left(e_{1}+\left[-e_{2}\right]\right) z^{n}=\frac{z-z^{3}}{1-4 x y z-\left(4\left(x^{2}+y^{2}\right)+2\right) z^{2}-4 x y z^{3}+z^{4}}
\end{aligned}
$$

from which we have the following theorem. 
Theorem 15. For $n \in \mathbb{N}$, the new generating functions of the product of Gaussian Pell polynomials is given by

$$
\begin{aligned}
& \sum_{n=0}^{+\infty} G P_{n}(x) G P_{n}(y) z^{n} \\
& \quad=\frac{-1+(4 x y+1) z+\left(4\left(x^{2}+y^{2}\right)+2 i(x+y)+1\right) z^{2}+(2 i(x+y)+4 x y-1) z^{3}}{1-4 x y z-\left(4\left(x^{2}+y^{2}\right)+2\right) z^{2}-4 x y z^{3}+z^{4}} .
\end{aligned}
$$

Proof. We know that

$$
G P_{n}(x)=i S_{n}\left(a_{1}+\left[-a_{2}\right]\right)+(1-2 i x) S_{n-1}\left(a_{1}+\left[-a_{2}\right]\right), \quad(\text { see }[13]) .
$$

and from it we obtain

$$
\begin{gathered}
\sum_{n=0}^{+\infty} G P_{n}(x) G P_{n}(y) z^{n}=\sum_{n=0}^{+\infty}\left(i S_{n}\left(a_{1}+\left[-a_{2}\right]\right)+(1-2 i x) S_{n-1}\left(a_{1}+\left[-a_{2}\right]\right)\right) \\
\times\left(i S_{n}\left(e_{1}+\left[-e_{2}\right]\right)+(1-2 i y) S_{n-1}\left(e_{1}+\left[-e_{2}\right]\right)\right) z^{n} \\
=i^{2} \sum_{n=0}^{+\infty} S_{n}\left(a_{1}+\left[-a_{2}\right]\right) S_{n}\left(e_{1}+\left[-e_{2}\right]\right) z^{n} \\
+i(1-2 i y) \sum_{n=0}^{+\infty} S_{n}\left(a_{1}+\left[-a_{2}\right]\right) S_{n-1}\left(e_{1}+\left[-e_{2}\right]\right) z^{n} \\
+i(1-2 i x) \sum_{n=0}^{+\infty} S_{n}\left(e_{1}+\left[-e_{2}\right]\right) S_{n-1}\left(a_{1}+\left[-a_{2}\right]\right) z^{n} \\
+(1-2 i x)(1-2 i y) \sum_{n=0}^{+\infty} S_{n-1}\left(a_{1}+\left[-a_{2}\right]\right) S_{n-1}\left(e_{1}+\left[-e_{2}\right]\right) z^{n} .
\end{gathered}
$$

By using the relationships (4.3) and (4.4), we obtain

$$
\begin{gathered}
\sum_{n=0}^{+\infty} G P_{n}(x) G P_{n}(y) z^{n}=\frac{z^{2}-1}{1-4 x y z-\left(4\left(x^{2}+y^{2}\right)+2\right) z^{2}-4 x y z^{3}+z^{4}} \\
+\frac{i(1-2 i y)\left(2 x z+2 y z^{2}\right)}{1-4 x y z-\left(4\left(x^{2}+y^{2}\right)+2\right) z^{2}-4 x y z^{3}+z^{4}} \\
+\frac{i(1-2 i x)\left(2 y z+2 x z^{2}\right)}{1-4 x y z-\left(4\left(x^{2}+y^{2}\right)+2\right) z^{2}-4 x y z^{3}+z^{4}} \\
+\frac{(1-2 i x)(1-2 i y)\left(z-z^{3}\right)}{1-4 x y z-\left(4\left(x^{2}+y^{2}\right)+2\right) z^{2}-4 x y z^{3}+z^{4}} \\
=\frac{-1+(4 x y+1) z+\left(4\left(x^{2}+y^{2}\right)+2 i(x+y)+1\right) z^{2}+(2 i(x+y)+4 x y-1) z^{3}}{1-4 x y z-\left(4\left(x^{2}+y^{2}\right)+2\right) z^{2}-4 x y z^{3}+z^{4}}
\end{gathered}
$$

The proof is completed. 


\section{Conclusion}

In this paper, by making use of Eq. (3.1), we have derived some new generating functions for the products of Gaussian Fibonacci numbers, Gaussian Lucas numbers, Gaussian Pell numbers, Gaussian Pell Lucas numbers, Gaussian Jacobsthal numbers, Gaussian Jacobsthal polynomials, Gaussian Jacobsthal Lucas polynomials and Gaussian Pell polynomials. The derived theorems are based on symmetric functions and products of these numbers and polynomials.

Acknowledgements. The authors are indebted to the anonymous referees for valuable comments and suggestions. Authors are supported by Directorate General for Scientific Research and Technological Development (DGRSDT), Algeria.

\section{REFERENCES}

[1] Abderrezzak, A., Généralisation de la transformation d'Euler d'une série formelle, Adv. Math., 103 (1994), 180-195.

[2] Abderrezzak, A., Généralisation d'identities de Carlitz, Howard et Lehmer. Aequ. Math., 49 (1995), 36-46.

[3] Boussayoud, A., Boughaba, S., Kerada, M., Araci, S., Acikgoz, M., Symmetric Functions of Binary Products of k-Fibonacci and Orthogonal Polynomials, Rev. R. Acad. Cienc. Exactas Fís. Nat, Ser. A Mat, RACSAM, 113 (2019), 2575-2586.

[4] Boussayoud, A., On some identities and generating functions for Pell-Lucas numbers, Online J. Anal. Comb., 12, (2017), 1-10.

[5] Boussayoud, A., Kerada, Boulyer, M. M., A simple and accurate method for determination of some generalized sequence of numbers, Int. J. Pure. Appl. Math., 108, (2016), 503-511.

[6] Boussayoud, A., Sahali, R., The application of the operator $L_{b_{1} b_{2}}^{-k}$ in the series $\sum_{j=0}^{+\infty} a_{j} b_{1}^{j} z^{j}$, J. Adv. Res. Appl. Math., 7, (2015), 68-75.

[7] Boussayoud, A., Kerada, M., Symmetric and Generating Functions, Int. Electron. J. Pure Appl. Math., 7, (2014), 195-203.

[8] Boussayoud, A., L'action de l'opérateur $\delta_{e_{1} e_{2}}^{k}$ sur la série $\sum_{n=0}^{+\infty} S_{n}(A) e_{1}^{n} z^{n}$, (Doctoral dissertation), Mohamed Seddik Ben Yahia University, Jijel, Algeria, (2017.

[9] Boussayoud, A., Boughaba,S., On Some Identities and Symmetric Functions for k-Pell sequences and Chebychev polynomials, Online J. Anal. Comb. 14 (2019), 1-13.

[10] Boussayoud, A., Kerada, M., Araci, S., Acikgoz, M., Esi, A., Generating Functions of Binary Products of Fibonacci and Tchebyshev Polynomials of first and second kinds, Filomat 33 (2019), 1495-1504.

[11] Djordjevic, G. B., Srivastava, H. M., Incomplete generalized Jacobsthal and Jacobsthal-Lucas numbers, Math. Comput. Modelling, 42 (2005), 1049-1056.

[12] Boubellouta, Kh., Boussayoud, A., Kerada, M., Symmetric Functions for Second-Order Recurrence Sequences, Tbil. Math. J., 13 (2020), 225-237.

[13] Boughaba, S., Boussayoud, A., Araci, S., Kerada, M., Construction of Generating Functions of Gaussian Fibonacci Numbers and Polynomials, Universal Journal of Mathematics and Applications, (Submitted).

[14] Jordan, J. H., Gaussian Fibonacci and Lucas numbers, Fibonacci Q., 3 (1965), 315-318.

[15] Asci, M., Gurel, E., Gaussian Jacobsthal and Gaussian Jacobsthal Lucas polynomials, Notes Number Theory Discrete Math., 19 (2013), 25-36.

[16] Halici, S., Oz, S., On Gaussian Pell Polynomials and Their Some Properties, Palest. J. Math., $7(1)(2018), 251-256$. 